\section{Principles and clinical application of ultrasound elastography for diffuse liver disease}

\author{
Woo Kyoung Jeong ${ }^{1}$, Hyo K. Lim ${ }^{1}$, Hyoung-Ki Lee², Jae Moon Jo², Yongsoo Kim³ \\ 'Department of Radiology and Center for Imaging Science, Samsung Medical Center, \\ Sungkyunkwan University School of Medicine, Seoul; ${ }^{2}$ Health Medical Equipment Business, \\ Samsung Electronics, Seoul; ${ }^{3}$ Department of Radiology, Hanyang University Guri Hospital, \\ Hanyang University College of Medicine, Guri, Korea
}

Accurate assessment of the degree of liver fibrosis is important for estimating prognosis and deciding on an appropriate course of treatment for cases of chronic liver disease (CLD) with various etiologies. Because of the inherent limitations of liver biopsy, there is a great need for noninvasive and reliable tests that accurately estimate the degree of liver fibrosis. Ultrasound (US) elastography is considered a non-invasive, convenient, and precise technique to grade the degree of liver fibrosis by measuring liver stiffness. There are several commercial types of US elastography currently in use, namely, transient elastography, acoustic radiation force impulse imaging, supersonic shear-wave imaging, and real-time tissue elastography. Although the low reproducibility of measurements derived from operator-dependent performance remains a significant limitation of US elastography, this technique is nevertheless useful for diagnosing hepatic fibrosis in patients with CLD. Likewise, US elastography may also be used as a convenient surveillance method that can be performed by physicians at the patients' bedside to enable the estimation of the prognosis of patients with fatal complications related to CLD in a non-invasive manner.

Keywords: Elasticity imaging techniques; Liver cirrhosis; Hypertension, portal; Ultrasonography

\section{Introduction}

Chronic liver disease (CLD) has various etiologies, with the viral infection of hepatitis B virus (HBV), hepatitis $\mathrm{C}$ virus (HCV), human immunodeficiency virus, alcohol consumption, hepatotoxic drug ingestion, non-alcoholic fatty liver, autoimmune diseases, and cryptogenic hepatopathy being commonly encountered in daily practice. Histologically, liver fibrosis develops and gradually progresses as a result of following a wound-healing response in patients with CLD. In particular, activation of cellular elements including myofibroblasts and stellate cells results in collagen deposition and subsequent development of CLD [1,2].

Liver biopsy is known as the gold standard for diagnosing liver fibrosis. However, liver biopsy also has considerable limitations. The very small size of samples obtained through biopsy may not represent a heterogeneous distribution of liver fibrosis due to sampling bias [3]. In addition, the

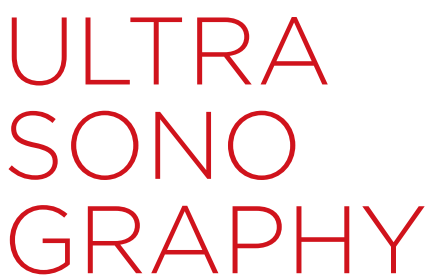

\section{REVIEW ARTICLE}

http://dx.doi.org/10.14366/usg. 14003 pISSN: 2288-5919 e elSSN: 2288-5943 Ultrasonography 2014;33:149-160

Received: January 9, 2014

Revised: March 9, 2014

Accepted: March 24, 2014

Correspondence to:

Hyo K. Lim, MD, Department of Radiology and Center for Imaging Science, Samsung Medical Center, Sungkyunkwan University School of Medicine, 81 Irwon-ro, Gangnam-gu, Seoul 135-710, Korea

Tel. +82-2-3410-2505

Fax.+82-2-3410-2559

E-mail: rfalim@skku.edu

This is an Open Access article distributed under the terms of the Creative Commons Attribution NonCommercial License (http://creativecommons.org/ licenses/by-nc/3.0/) which permits unrestricted noncommercial use, distribution, and reproduction in any medium, provided the original work is properly cited.

Copyright @ 2014 Korean Society of Ultrasound in Medicine (KSUM)

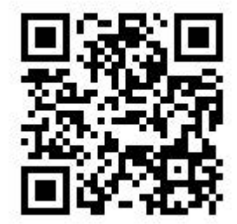

How to cite this article:

Jeong WK, Lim HK, Lee HK, Jo JM, Kim $Y$. Principles and clinical application of ultrasound elastography for diffuse liver disease. Ultrasonography. 2014 Jul;33(3):149160. 
issue of intra- or inter-observer variability among pathologists in evaluating the grade of fibrosis is an additional limitation because the interpretation process is a subjective and semi-quantified method [4]. According to previous research on chronic hepatitis $C$, agreement among pathologists regarding the fibrosis grade is not excellent $(\kappa$, about 0.5$)$ [5]. Although the rate of complications is very low and the risk has declined with the use of ultrasonographic guidance [6], liver biopsy is somewhat invasive and post-biopsy bleeding can be serious. With respect to non-invasive alternatives to liver biopsy, several serological or biochemical methods for the estimation of liver fibrosis have been validated primarily in patients with chronic hepatitis $C$, but still lack the ability to identify and classify the intermediate stages of fibrosis [7].

Introduced in 1991, elastography is another non-invasive technique for evaluating the elastic properties of soft tissue either quantitatively or qualitatively [8]. The elastography of the liver is theoretically not easy to determine compared with that of superficial organs because the liver is located deep and under the rib cage. Nevertheless, various techniques of ultrasound (US) elastography have been developed for repeatedly measuring hepatic fibrosis. From a technical standpoint, two types of US elastography for the measurement of liver stiffness are under development: shear wavebased elastography and real-time tissue elastography (Fig. 1, Table 1). This review addresses the principles and clinical usefulness of US elastography for the diffuse liver disease with an emphasis on shear wave-based elastography.

\section{Basic Principles of Elastography}

Elastography is a promising imaging technique because the elastic modulus of tissues measured by this technique provides the most broad-banded properties compared with other quantitative values measured by computed tomography (attenuation value), magnetic resonance (MR) imaging ( $\mathrm{T} 1$ relaxation time), and conventional ultrasonography (bulk modulus). The order of magnitude of the elastic modulus is approximately five times larger than that for other imaging modalities [9], meaning that the use of the elastic modulus can maximize the discrimination between different tissues or between normal tissue and lesions. The elastic modulus is defined as the slope of the stress-strain curve during elastic deformation. Therefore, a stiffer object has a higher elastic modulus. There are various approaches to elastic imaging, all of which consist of three basic steps: excitation (stress) application, tissue response (strain) measurement, and mechanical parameters estimation [9].

\section{Excitation Application}

In its most basic form, shear wave-based elastography applies a perpendicular stress force on the target organ to induce "shear" on the tissue (Fig. 2). By definition, shear is the change of shape (displacement)-without a change in volume-produced by a pair of forces acting in opposite directions. At this point, transversely propagating waves with a very low velocity develop in the tissue,

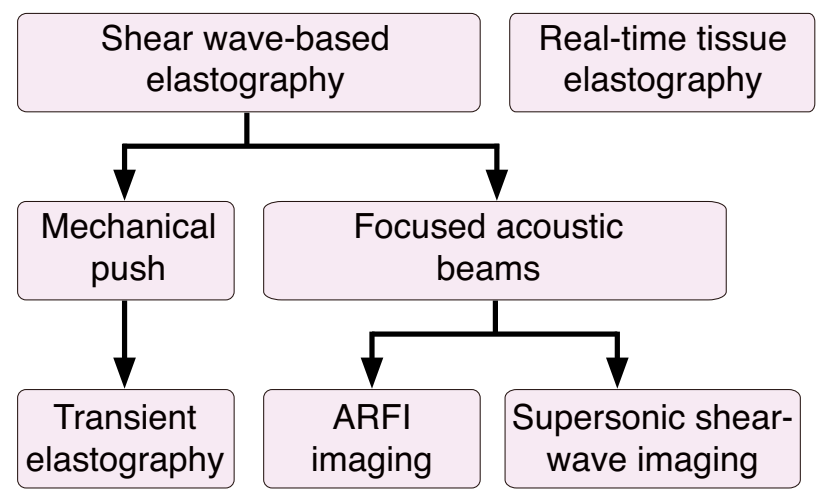

Fig. 1. Classification of ultrasound elastography of the liver. ARFI, acoustic radiation force impulse.

Table 1. Comparison of various techniques of ultrasound elastography

\begin{tabular}{|c|c|c|c|c|}
\hline Technique & Transient elastography & ARFI imaging & $\begin{array}{c}\text { Supersonic shear-wave } \\
\text { imaging }\end{array}$ & $\begin{array}{c}\text { Real-time tissue } \\
\text { elastography }\end{array}$ \\
\hline Type of force & Dynamic & Dynamic & Dynamic & Quasi-static \\
\hline Applied force & Mechanical impulse & $\begin{array}{l}\text { US-induced radiation force } \\
\text { impulse }\end{array}$ & $\begin{array}{l}\text { US-induced radiation force } \\
\text { impulse }\end{array}$ & Intrinsic (heartbeat) \\
\hline Measurement of strain & $\begin{array}{l}\text { Single measure, beam-line } \\
\text { average }\end{array}$ & Single image within a box & Image within a color box & Full area image \\
\hline Estimated parameter & $\begin{array}{l}\text { Elastic modulus converted from } \\
\text { shear wave velocity }(\mathrm{kPa})\end{array}$ & Velocity of shear wave $(\mathrm{cm} / \mathrm{sec})$ & $\begin{array}{l}\text { Elastic modulus converted from } \\
\text { shear wave velocity }(\mathrm{kPa})\end{array}$ & Strain ratio \\
\hline Qualitative or quantitative & Quantitative & Qualitative/quantitative & Quantitative & Qualitative \\
\hline Clinical evidence & Very much & Much & Little & Scanty \\
\hline
\end{tabular}


which are called shear waves. In the case of transient elastography, a mechanical push is used for excitation application, which produces transient shear waves in the target tissue. This type of excitation application is classified as a dynamic elastography technique with an external source. In addition, dynamic techniques induce vibrations and comprise the basic method for shear wave-based US elastography and MR elastography. In particular, rather than a mechanical push with transient elastography, focused US beams from a US transducer make shear waves through the absorption of acoustic energy; acoustic radiation force impulse (ARFI) imaging and supersonic shear-wave imaging (SSI) belong to this category [10]. With respect to shear wave elastography (Supersonic Imagine, Aix-en-Provence, France), a type of SSI, multiple acoustic radiation forces are successively focused at different depths in a tissue to generate a strong shear wave to propagate along the tissue at a safe level of acoustic power, which is then coherently summed in a Mach cone shape and improves the propagation distance [11]. On the other hand, real-time tissue elastography methods are derived from the static elastography technique used for the measurement of breast tissue elasticity, and employ the quasi-static or intrinsic stress derived from heartbeats $[12,13]$.

\section{Tissue Response Measurement}

Measurement of tissue response is the most critical component of elastography. The basic measurement method consists of a comparison of successively obtained images and a reference image (Fig. 2). Through either a mechanical push or acoustic radiation force, the A-axis (direction of force=depth direction) displacement of the target tissue occurs, and the shear waves are generated simultaneously. These are very slow $(1-10 \mathrm{~m} / \mathrm{sec})$ compared with an US beam and travel perpendicular to the direction of the stress force. To detect a shear wave, two methods using US have been introduced. Transient elastography causes a single transient shear wave to propagate along the $\mathrm{A}$-axis direction by using an $\mathrm{M}$-mode US technique and calculates Young modulus of the tissue by using this information [14]. Another method is the Doppler technique, in which radiofrequency (RF) images including the information of the propagating shear waves are measured using the echo of the transmitted US beams at a very high frame rate, which can be used to generate a tissue displacement map $[8,15,16]$. Using the tissue displacement maps obtained during the period of shear wave propagation (i.e., less than $14 \mathrm{~ms}$ ), it is possible to calculate the velocity of a shear wave by analyzing the movement of the peak of the shear wave. In this way, the elastic modulus can be calculated by $E=3 \rho V_{s}{ }^{2}$ where $\rho$ denotes the density of the tissue and $V_{s}$ represents the velocity of the shear wave.

\section{Mechanical Parameter Estimation}

Both qualitative and quantitative methods are used to perform mechanical parameter estimation (Fig. 2). Liver stiffness is usually

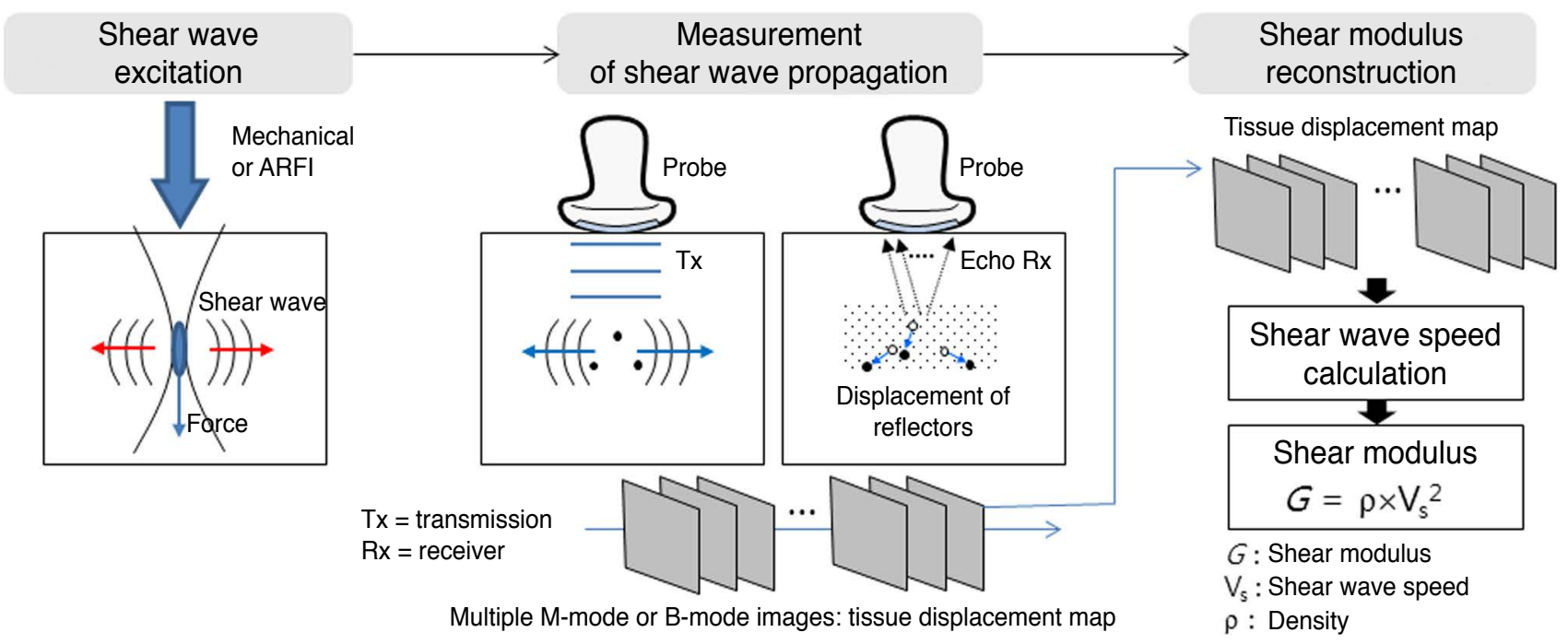

Fig. 2. Diagram depicting the process of shear wave-based ultrasound elastography. Shear wave-based elastography applies a perpendicular stress force to a target organ in order to induce shear on the tissue. The information on the propagating shear wave including the velocity of the shear wave could be measured by obtaining radiofrequency images with a high frame rate, which can be used to generate a tissue displacement map. Then, the elastic property for quantitative estimation is calculated by the propagating velocity of the shear wave. ARFI, acoustic radiation force impulse. 
measured quantitatively. The elastic properties for quantitative estimation are expressed as Young modulus (E) or the shear modulus $(\mu)$. For most soft tissues, Young modulus and the shear modulus are related by a simple scale factor of 3: i.e., $\mathrm{E}=3 \mu$ [9]. Transient elastography and SSI display tissue stiffness as the elastic modulus ( $\mathrm{kPa}$ ), while ARFI imaging shows it by shear wave velocity $(\mathrm{cm} / \mathrm{sec})$. In the case of real-time elastography, tissue elasticity cannot be measured directly from the reflected US echo. Relative tissue elasticity is thus calculated and displayed as a color overlay of the conventional B-mode image, and the strain ratio between two different points can be obtained instead of elastic modulus or shear wave velocity.

\section{Methods of Shear Wave-based Elastography}

\section{Transient Elastography}

Transient elastography was the first commercialized elastography method developed to noninvasively assess the stiffness of deep soft tissues such as the liver. Transient elastography consists of two parts: a mechanical vibrator and a single-channel US transducer [14]. The mechanical vibrator generates a low-frequency wave at $50-500 \mathrm{~Hz}$ to generate shear stress in the target tissue at a length of $4 \mathrm{~cm}$, and the velocity of the shear wave can then be measured using an US signal (Fig. 3). The most superior advantage of transient elastography is that it has been extensively validated by numerous investigations targeted at patients with CLD, and the results of transient elastography are generally accepted to be well correlated with different stages of liver fibrosis. The validity of liver stiffness measurements are determined by the success rate and the interquartile range divided by the median (IQR/M) in cases with more than 10 valid measurements. Here, the success rate is the ratio of the number of valid measurements to the total number of measurements and should be greater than 60\%; IQR/M should be lower than 30\% [17]. Despite these advantages, there are several disadvantages of transient elastography. They are as follows: first, transient elastography does not provide a B-mode image, which is essential for accurate targeting. Second, transient elastography is not performed for the patient with ascites. In addition, transient elastography exhibits a relatively high measurement failure rate of $4.5 \%-6 \%$. Major factors related to this failure rate include a body mass index greater than 28 , age over 50 years, non-alcoholic steatohepatitis, diabetes, and a $\gamma$-glutamyl-transpeptidase level higher than $57 \mathrm{IU} / \mathrm{L}$ [18].

\section{Acoustic Radiation Force Impulse Imaging and Shear Wave Imaging}

Both ARFI imaging and SSI use focused high-intensity, shortduration acoustic pulses instead of the mechanical vibration of transient elastography in order to produce shear waves in the target tissue [19]. ARFI imaging generates shear waves by a single pushing beam, while shear wave propagation is monitored using conventional pulse-echo US at multiple off-axis lateral locations. By collecting displacement through time information at multiple lateral locations separated by a known distance from the excitation source, the speed of the propagating shear waves can be estimated (Fig. 4). The region of interest (ROI) of ARFI imaging is relatively small (i.e.,

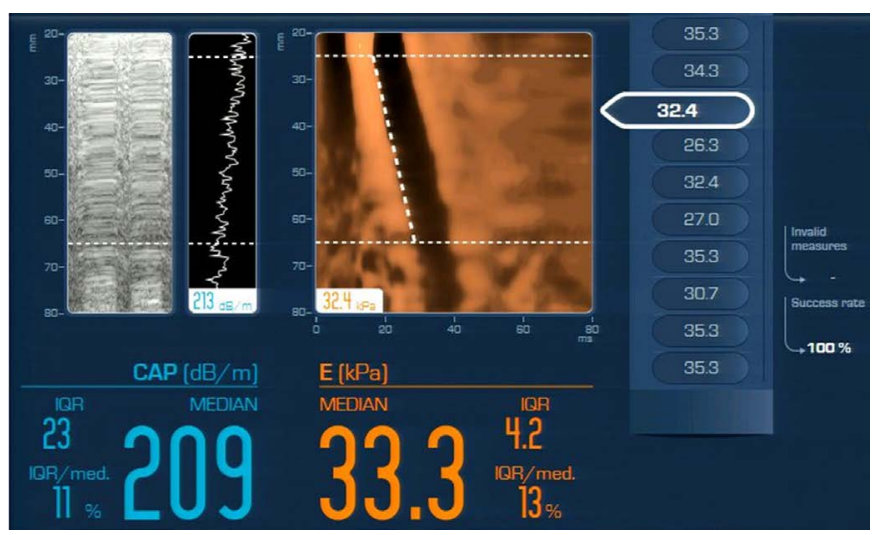

B

A

Fig. 3. Transient elastography of a normal and a cirrhotic patient.

The displacement M-mode image located in the center of the monitor shows axial displacement as a function of depth ( $y$-axis) and time (x-axis). In the healthy patient (A), the shear wave is relatively slow and the liver stiffness is low. However, in the patient with cirrhosis (B), the shear wave can propagate more rapidly though a hard tissue, and the time-depth gradient is very steep. The controlled attenuation parameter is displayed, which is known to correlate with the fatty liver severity (Courtesy of Dr. Yong Han Paik, Department of Internal Medicine, Samsung Medical Center, Seoul, Korea). 
$10 \mathrm{~mm} \times 5 \mathrm{~mm}$ ) as compared to that of SSI.

SSI is a new shear wave-based US elastography technique. SSI generates push beams at multiple axial depths to create a nearsupersonic plane wave shear and transmits the unfocused beam (plane wave) to monitor the shear wave propagation throughout the ROI (Fig. 5). The ROI of SSI is fan-shaped and larger than other modalities (up to $50 \mathrm{~mm} \times 50 \mathrm{~mm}$ ) $[20,21]$. A remarkable feature of SSI is that it can show viscoelastic properties in all areas in an ROI with a color look-up table and thus, is expected to overcome the limitations of transient elastography by which liver stiffness cannot be measured accurately in patients with severe obesity, thick subcutaneous fat, and ascites [22]. Moreover, the same technique can be used to display a grayscale US image on the background of the elastogram, so it is more reliable and familiar to a physician who uses conventional ultrasonography. However, for as wide a use of SSI for the diagnosis of liver fibrosis as that of transient elastography, further clinical experiences and evidence are needed.

\section{Real-Time Tissue Elastography}

As mentioned above, real-time elastography is a method derived from the static elastography technique used for the measurement of breast tissue elasticity. Real-time tissue elastography uses a grayscale US machine, incorporating elastography into the conventional US scanner. This approach uses a quasi-static method

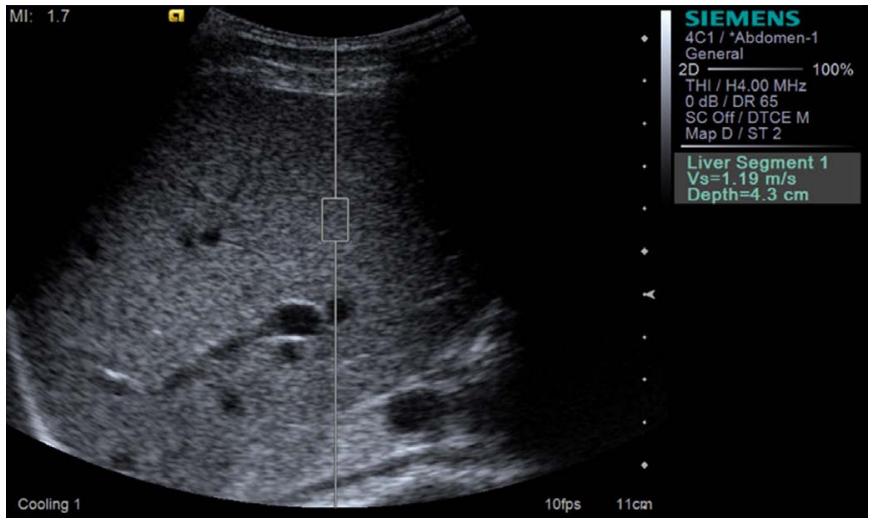

A

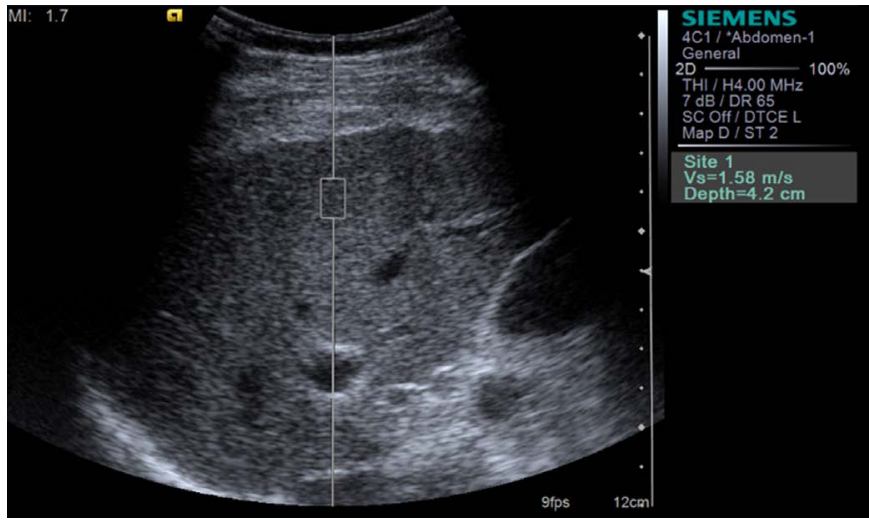

B

Fig. 4. Acoustic radiation force impulse imaging of a normal and a cirrhotic patient.

The cylindrical region of interest (ROI) in the middle of the ultrasonogram was used as the sample volume for the measurement of stiffness. Instead of Young modulus, the propagating velocity of the shear wave is displayed. Although the grayscale ultrasonograms are similar to each other, the propagating velocity is different: $1.2 \mathrm{~m} / \mathrm{sec}$ in the healthy patient (A) and $1.6 \mathrm{~m} / \mathrm{sec}$ in the patient with cirrhosis (B) (Courtesy of Dr. Yong Eun Chung, Department of Radiology, Severance Hospital, Seoul, Korea).

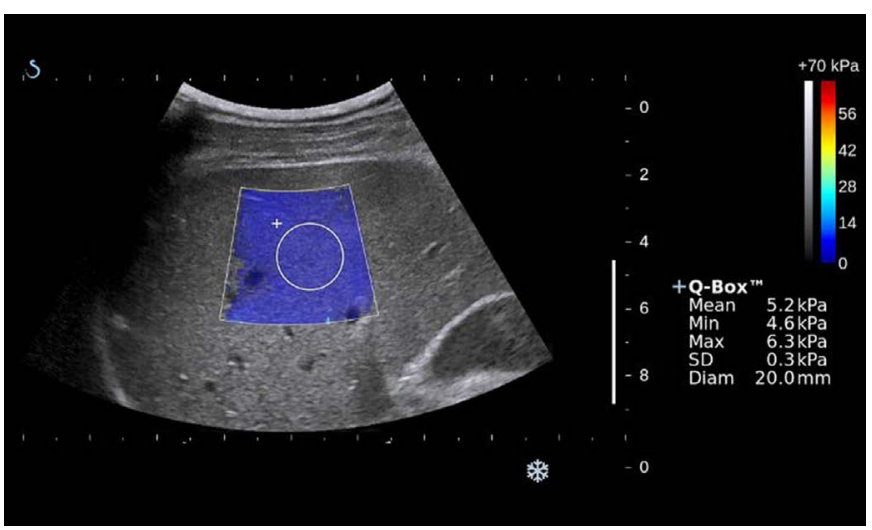

A

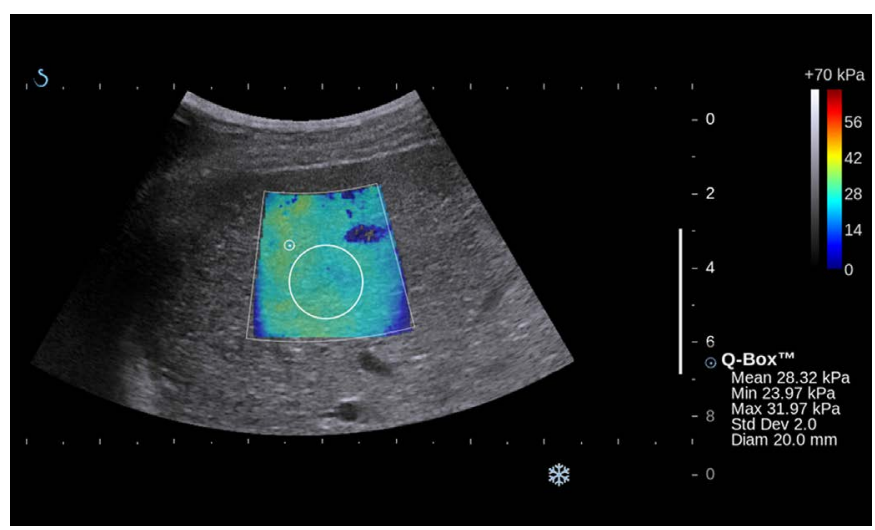

B

Fig. 5. Supersonic shear-wave imaging of a normal and a cirrhotic patient.

A large trapezoidal color box display of the distribution of the elastic properties of the liver. The severity of stiffness is depicted with the colored look-up table. Dark blue color represents normal liver tissue (A), and bright blue-green color represents increased liver stiffness such as liver cirrhosis (B). The round region of interest (ROI) in the color box is the Q-Box and the mean Young modulus and standard deviation in the ROI have been calculated. 
consisting of an excitation application in which the examiner has to compress and relax the body by a transducer or utilize intrinsic stress derived from the heartbeat $[12,13]$. Simultaneously, echo signals are captured in real time, and the relative stiffness (elastic ratio) of the liver can be calculated along with a real-time color map. Like SSI, real-time tissue elastography can display tissue elasticity images and conventional grayscale US images at the same time but is unable to calculate the elastic modulus (Fig. 6).

\section{Clinical Applications of US Elastography}

\section{Hepatic Fibrosis}

In terms of the diagnostic performance of US elastography for measuring liver stiffness, there have been many clinical studies regarding the diagnosis of hepatic fibrosis using transient elastography $[23,24]$. According to a recent meta-analysis aimed at chronic hepatitis $C$ patients, the pooled estimate of the cut-off value for significant fibrosis ( $\geq \mathrm{F} 2$ on the METAVIR score system) was $7.71 \mathrm{kPa}$ with a sensitivity of $72 \%$ and specificity of $82 \%$. In the case of cirrhosis (F4), the results showed a cut-off of $15.08 \mathrm{kPa}$ with a sensitivity of $84 \%$ and specificity of $95 \%$. Another meta- analysis of 40 eligible studies showed that the summary sensitivity and specificity were $78 \%$ and $80 \%$ for significant fibrosis, and $83 \%$ and $90 \%$ for cirrhosis, respectively (Table 2). In addition, this metaanalysis suggested that transient elastography could be used as a good screening test for cirrhosis, but not for accurately diagnosing fibrotic stages other than cirrhosis because no optimal cut-offs of liver stiffness for individual fibrosis stages have been validated. There are relatively fewer clinical studies of ARFI imaging and SSI compared with transient elastography studies, although some studies showed that the performance and reliability of ARFI imaging and SSI are comparable to those of transient elastography $[19,20,25]$. According to a recent meta-analysis study for ARFI imaging, the mean diagnostic accuracy of ARFI expressed as areas under the receiver operating characteristic curves (AUROCs) was 0.87 for $\geq F 2,0.93$ for $\geq F 3$, and 0.93 for $F 4$ [26]. In the case of real-time elastography, the elastic ratio (ratio of the value in the intrahepatic venous small vessels to the value in the parenchyma) was calculated instead of liver stiffness, and the diagnostic performance of this technique was superior to that of non-invasive biochemical markers including aspartate aminotransferase to the platelet ratio index (APRI) and the Forns Index [12].

Table 2. Summary sensitivity, specificity, and diagnostic odds ratio (DOR) to diagnose F2 and F4 fibrosis by using transient elastography extracted from a meta-analysis

\begin{tabular}{ccccccc}
\hline Causes & Stage & Studies & Cut-off liver stiffness & Sensitivity (\%) & Specificity (\%) & DOR \\
\hline Chronic hepatitis C & $\geq F 2$ & 14 & $7.6(5.1-10.1)$ & $78(71-84)$ & $80(71-86)$ & 13.9 \\
& F4 & 11 & $15.3(11.9-26.5)$ & $83(77-88)$ & $90(87-93)$ & 46.5 \\
Chronic hepatitis B & $\geq$ F2 & 4 & $7.0(6.9-7.2)$ & $84(67-93)$ & $78(68-85)$ & 17.9 \\
& F4 & 6 & $11.3(9.0-13.4)$ & $80(61-91)$ & $89(82-94)$ & 34.3 \\
\hline
\end{tabular}

Modified from Tsochatzis et al. [24]

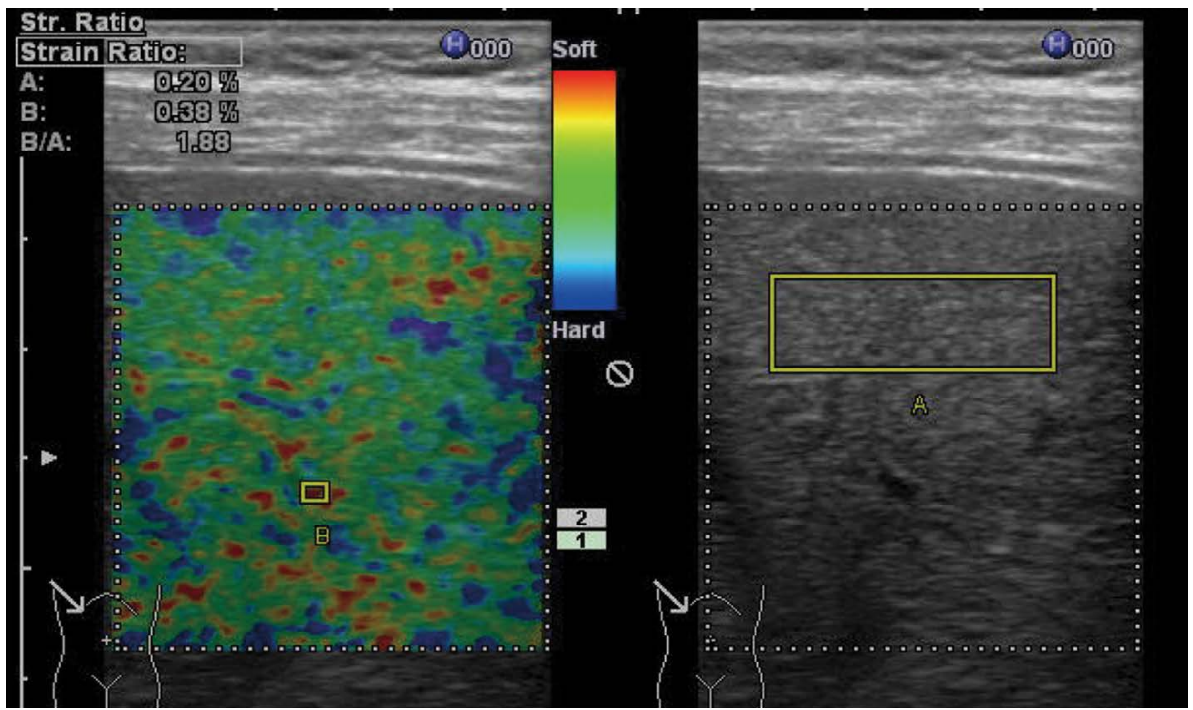

Fig. 6. Real-time tissue elastography of the liver. The elastic ratio calculated from the tissue compressibility of the liver (A on the right) and that of the intrahepatic small vessel ( $B$ on the left) represents liver stiffness. On the look-up table, red and blue indicate soft and hard tissues, respectively. Reprinted from Koizumi et al. [12] with permission from the Radiological Society of North America. 
The diagnostic performance of US elastography in patients with an HBV infection has not yet been well addressed. Marcellin et al. [27] first reported the diagnostic accuracy of transient elastography in the patients with chronic hepatitis B. Although the clinical evidence for fibrosis staging in patients with chronic hepatitis $B$ is relatively weak and the overall diagnostic performance is slightly worse than for chronic hepatitis $C$ patients, the diagnostic accuracy of transient elastography is acceptable [28]. In a recent meta- analysis of the performance of transient elastography for the staging of liver fibrosis in chronic hepatitis B, the mean AUROCs for the diagnosis of significant fibrosis, severe fibrosis, and liver cirrhosis were $0.859,0.887$, and 0.929 , respectively, as determined from 18 studies comprising 2,772 patients [29]. Compared with HCV patients, the cut-off value for cirrhosis in HBV patients tends to be lower because the liver fibrosis area in macronodular cirrhosis is relatively smaller. In contrast, alanine aminotransferase flares can

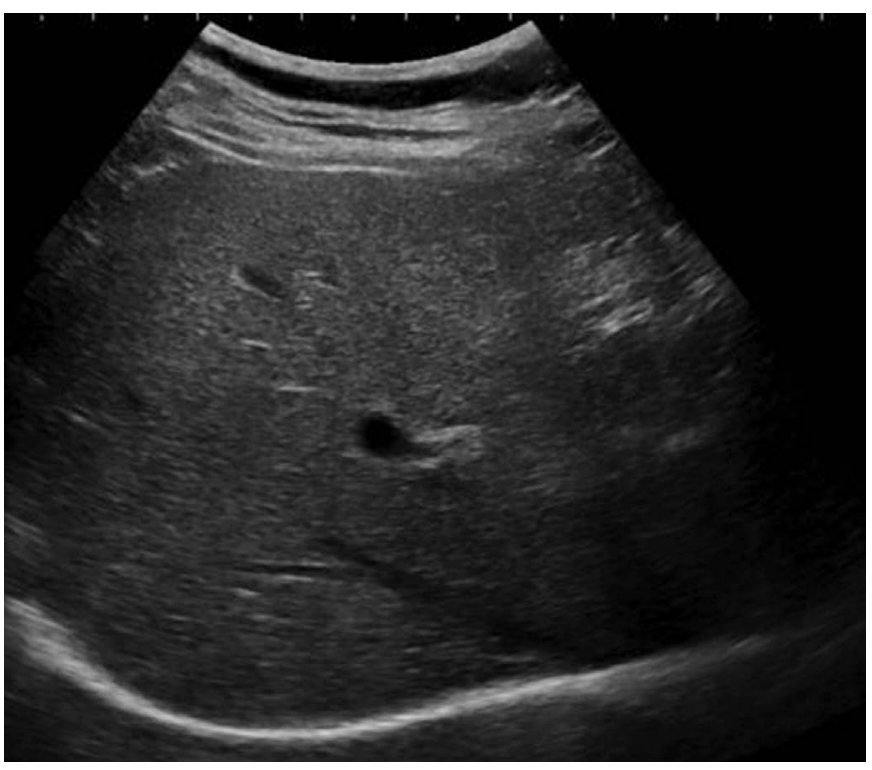

A

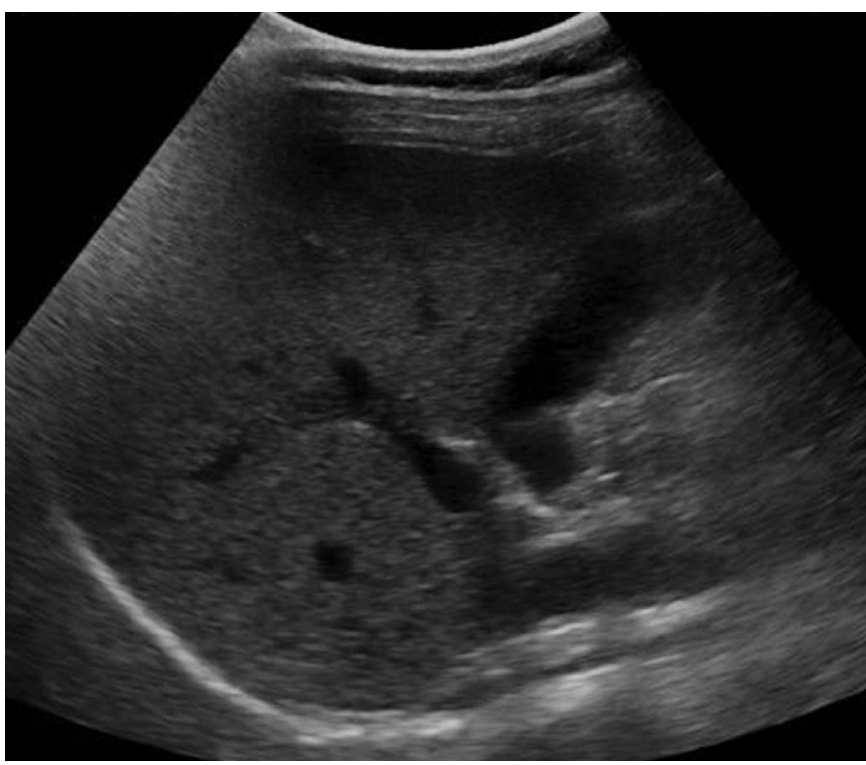

C

Fig. 7. Acute exacerbation of hepatitis B virus-induced hepatitis.

Grayscale ultrasonography (A) shows mild parenchymal coarseness of the liver; the liver stiffness is $10.3 \mathrm{kPa}$ according to the shear wave elastography (B). Six months later, the patients recovered from liver function deterioration. The grayscale ultrasonographic feature (C) is similar to the previous study, but the liver stiffness decreases to $6 \mathrm{kPa}$ (D). 
result in overestimating the fibrosis grade, and thus, the increased liver stiffness should be interpreted carefully in patients with acute exacerbation of chronic HBV hepatitis [30] (Fig. 7).

\section{Non-Alcoholic Fatty Liver Disease}

Non-alcoholic fatty liver disease (NAFLD) actually represents an emerging disease of great clinical interest because of the increasing incidence of metabolic diseases and obesity in recent decades. The disease spectrum of NAFLD is very wide, ranging from simple fatty liver to non-alcoholic steatohepatitis, and liver fibrosis can develop and progress to liver cirrhosis [31]. Although transient elastography is difficult to perform in cases of obesity because subcutaneous fatty tissue attenuates the pushing pulse, its role in causing NAFLD has recently been highlighted owing to the development of a new technique utilizing a vibration-controlled transient elastography device, which allows the calculation of the new controlled attenuation parameter (CAP). CAP is known to be useful for the non-invasive and accurate estimation of liver steatosis [32]. In addition, a new XL probe designed to measure shear waves at deeper positions by using a lower central US frequency ( 2.5 $\mathrm{MHz}$ ) can be applied, thereby allowing more reliable results to be obtained compared with conventional M probes [33]. According to a diagnostic algorithm introduced by a recent review article [34], transient elastography could be useful to stratify patients at indeterminate risk; if liver stiffness was lower than $7.9 \mathrm{kPa}$, advanced fibrosis could be excluded with a $97 \%$ negative predictive value. On the other hand, if liver stiffness was higher than $9.6 \mathrm{kPa}$, the diagnosis of advanced fibrosis could be made with a $72 \%$ positive predictive value [35]. Thus, only the remaining patients (7.9-9.6 kPa) require liver biopsy for diagnosis, and the number of NAFLD patients with the indication of liver biopsy can be decreased, leading to a reduced incidence of biopsy-induced complications [34].

\section{Follow-up after Liver Transplantation}

After liver transplantation, US elastography is also useful to diagnose liver fibrosis caused by relapsed chronic hepatitis and the acute rejection of the liver graft. A systematic review of studies comparing US elastography to liver biopsy for the detection of liver fibrosis by a recurrent HCV infection stated that the diagnostic accuracy for significant fibrosis (F2) using transient elastography was generally good, with a sensitivity and a specificity of $83 \%$. Further, with respect to liver cirrhosis, the sensitivity and specificity were improved to $98 \%$ and $84 \%$, respectively [36]. Liver stiffness may also be increased by acute cellular rejection following liver transplantation; however, it is important to keep in mind that liver stiffness can increase in transplanted livers without evidence of rejection since it may undergo ischemic or reperfusion injury within 4 weeks from transplantation, which can in turn result in transient hepatocellular ballooning and hepatocanalicular cholestasis with inflammation that may recover within 2-3 weeks without specific treatment. Therefore, US elastography may be useful to detect rejection at follow-up more than 4 weeks after transplantation [37].

\section{Portal Hypertension}

Estimation of the severity of portal hypertension in patients with liver cirrhosis is another major use of liver stiffness measurements. Increased portal pressure is the major factor driving the clinical course of cirrhosis. Measurement of the hepatic venous pressure gradient (HVPG) following hepatic venous catheterization was used as a surrogate marker of portal hypertensive stigmata. Recently, there were some investigations concerning the feasibility of the noninvasive measurement of liver stiffness to estimate severe portal hypertension $[38,39]$. Looking at the results of these studies, we concluded that liver stiffness could be closely correlated with both HVPG and the presence of complications related with portal hypertension (Fig. 8).

\section{Roles for Longitudinal Surveillance}

To date, the majority of elastography studies have focused on evaluating the cross-sectional performance with respect to the histological fibrosis grade or HVPG. However, an important but undervalued use of elastography is the ability to repeatedly measure liver stiffness. The roles of elastography as longitudinal perspectives with respect to the prediction of the long-term prognosis of the disease and monitoring of clinical courses with or without treatment are well known. In particular, these approaches can be used to non-invasively estimate the prognosis of the patients with fatal complications related to CLD, such as variceal bleeding and decompensation.

A longitudinal follow-up of elastography has been proposed as a way to establish the tailored management strategies by providing more detailed prognostic information [40]. For example, the concept of cirrhosis has recently changed from dynamic to bidirectional. In other words, cirrhosis patients may recover if antiviral therapy can be applied properly. At this time, the ideal approach to assess histological outcomes during treatment is serial liver biopsy; however, this is not possible in most cases. Instead, the measurement of liver stiffness by elastography is very useful for monitoring the changes in liver fibrosis during the antiviral treatment $[41,42]$. In terms of portal hypertension, elastography may also be used to predict the development of variceal bleeding by using a hybrid parameter, the liver stiffness-spleen diameter to platelet ratio score (LSPS) defined as the product of liver stiffness and the maximum spleen diameter divided by the platelet count [43]. According to risk stratification 


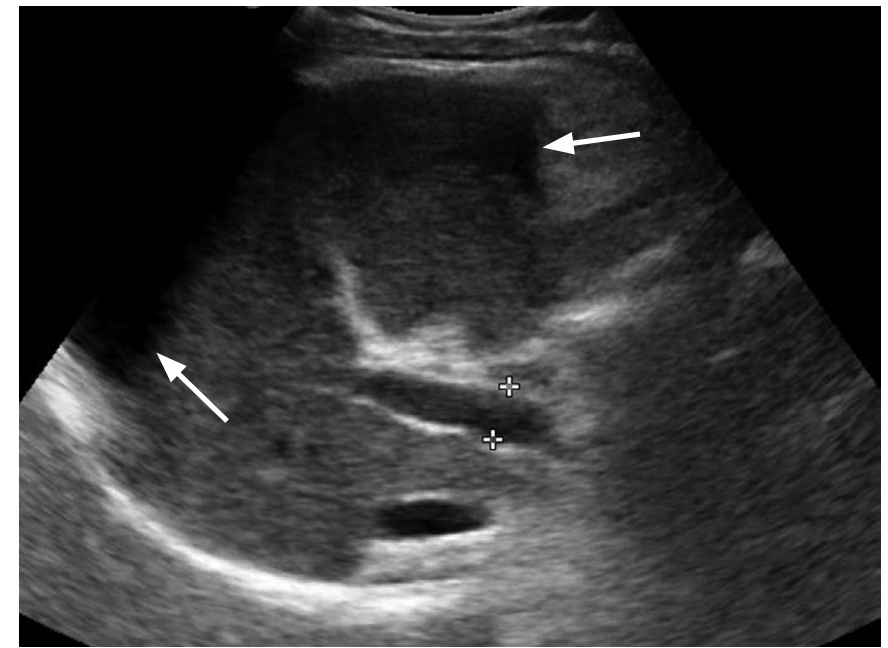

A

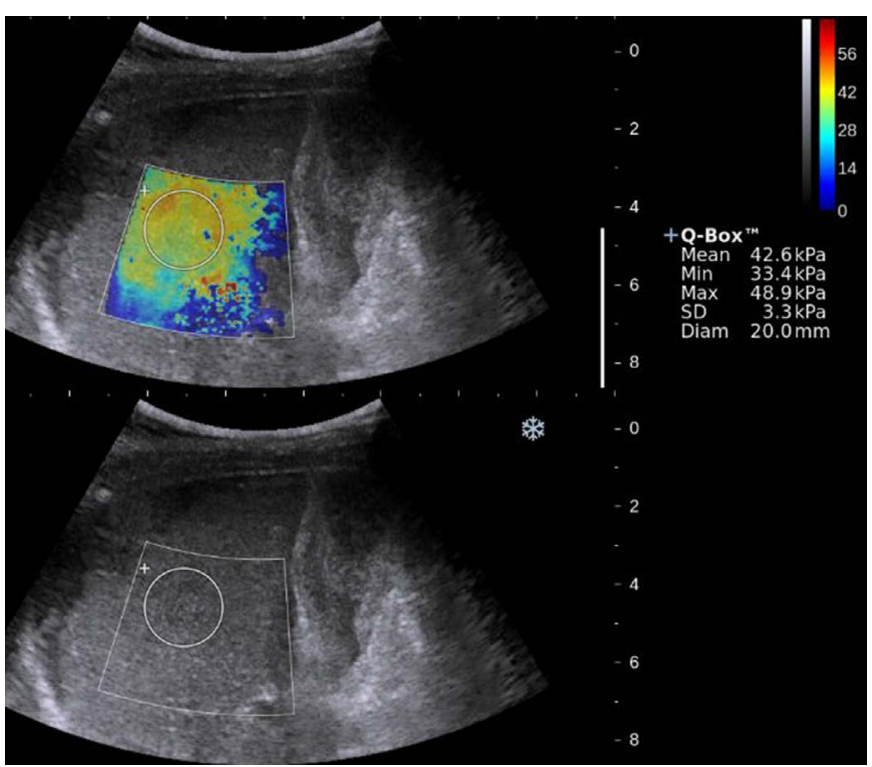

B

based on LSPS, a different prophylactic treatment for the prevention of variceal bleeding should be considered for patients with an LSPS value higher than 6.5 points [44].

\section{US Elastography: Weaknesses and Strengths}

The most significant challenge facing US elastography is the issue of measurement reproducibility. A number of studies concerning this issue have been published; however, many investigators have brought up questions about this issue due to the inherent limitations of US such as the operator-dependent performance. Transient elastography is a highly reproducible and user-friendly technique [45], and liver stiffness measurement by transient elastography does not require a learning curve: even a novice can obtain a reliable

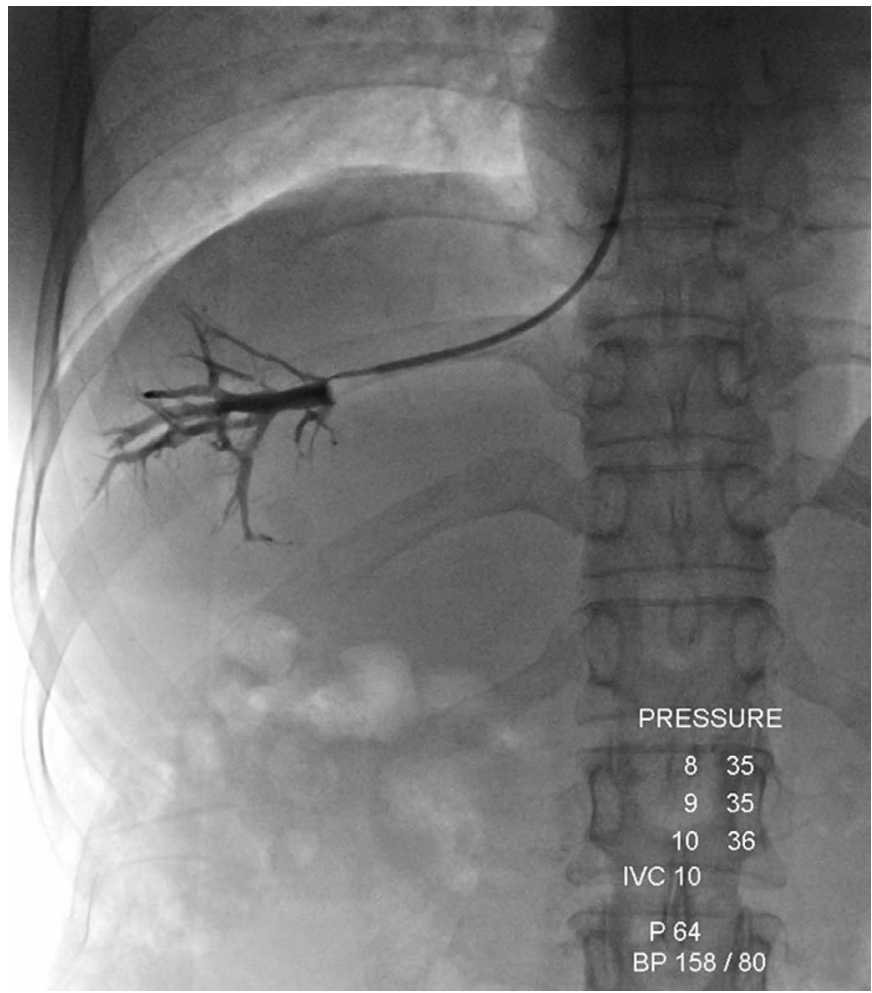

C

Fig. 8. Shear wave elastography of a patient with portal hypertension.

On grayscale ultrasonography, the cirrhotic change of the liver and the perihepatic ascites (arrows) are noted (A). The measured liver stiffness is $42.6 \mathrm{kPa}(\mathrm{B})$, which is about 8 times the normal value. The hepatic venous pressure gradient was $26.3 \mathrm{mmHg}$, which indicates severe portal hypertension (C).

result after a single training session [46]. However, because liver stiffness measurements can be influenced significantly by steatosis, obesity, lower degrees of hepatic fibrosis [45], necroinflammation of hepatocytes [47], cholestasis [48], elevated central venous pressure [49], and even postprandial conditions [50], it should be carefully applied when used as an alternative measurement of liver stiffness instead of liver biopsy.

In the case of ARFI, the overall reproducibility is also not bad, having an intraclass correlation coefficient (ICC) value for the interrater observation of 0.81 and an ICC for the intrarater observation of 0.90 . However, gender (women), high body mass index, ascites, and lower degree of liver disease (noncirrhotic patients) are considered factors that impede the reproducibility of ARFI [51]. In the case of SSI, the inter- and intraobserver agreements 
have ICC values of 0.88 and 0.94 , respectively, which are similar to the results of ARFI imaging [52].

Despite the issues described above, US elastography has many advantages in clinical fields. The most important aspect is convenience, as is the case with most ultrasonography examination techniques. Indeed, US elastography is fast, easy to use, and portable, so much so that it can be performed at the patient's bedside. Likewise, because it does not use ionizing radiation, US elastography is relatively safe, even in patients who repeatedly undergo the procedure. US elastography is also less expensive than MR elastography [53]. Going forward, the most important strength of US elastography is the availability of a large amount of accumulated clinical data that have demonstrated its clinical usefulness, although most of these data are related to transient elastography.

\section{Conclusions}

Measurement of liver stiffness using various technical developments is evolving to overcome its limitations. Recently, the European Federation of Societies for Ultrasound in Medicine and Biology (EFSUMB) published an informative guideline for the use of US elastography $[54,55]$ that deals with the relevant technology and clinical applications. Along with the basic principles for use, these guidelines include the practical advantages and disadvantages of US elastography as well as recommendations for the examination of various body parts. According to these guidelines, US elastography is useful to assess the severity of liver fibrosis in patients with diffuse liver disease and particularly to distinguish patients with nil to mild fibrosis from those with significant fibrosis, although some of the newer techniques must be validated through clinical studies. At present, however, US elastography for the differentiation of focal hepatic lesions is not recommended.

In conclusion, US elastography is useful for diagnosing hepatic fibrosis in patients with CLD and may be used as a convenient and non-invasive surveillance method to estimate the prognosis of patients with fatal complications related to CLD. Accordingly, the development of a standardized method for liver stiffness measurement and technical improvements should be a priority for the clinical application of US elastography. Together, these efforts will significantly enhance the clinical implications of US elastography.

ORCID: Woo Kyoung Jeong: http://orcid.org/0000-0002-0676-2116; Hyo K. Lim: http://orcid.org/0000-0003-3269-7503; Hyoung-Ki Lee: http://orcid.org/0000-00017206-2081; Jae Moon Jo: http://orcid.org/0000-0003-3096-0558; Yongsoo Kim: http://orcid.org/0000-0002-1069-0135

\section{Conflict of Interest}

No potential conflict of interest relevant to this article was reported.

\section{Acknowledgments}

This paper was supported by the research fund from Samsung Medison, Inc.

\section{References}

1. Friedman SL. Mechanisms of hepatic fibrogenesis. Gastroenterology 2008;134:1655-1669.

2. Talwalkar JA. Elastography for detecting hepatic fibrosis: options and considerations. Gastroenterology 2008;135:299-302.

3. Bedossa P, Dargere D, Paradis V. Sampling variability of liver fibrosis in chronic hepatitis C. Hepatology 2003;38:1449-1457.

4. The French METAVIR Cooperative Study Group. Intraobserver and interobserver variations in liver biopsy interpretation in patients with chronic hepatitis C. Hepatology 1994;20:15-20.

5. Gronbaek K, Christensen PB, Hamilton-Dutoit S, Federspiel BH, Hage $E$, Jensen $0 J$, et al. Interobserver variation in interpretation of serial liver biopsies from patients with chronic hepatitis C. J Viral Hepat 2002;9:443-449.

6. Al Knawy B, Shiffman M. Percutaneous liver biopsy in clinical practice. Liver Int 2007;27:1166-1173.

7. Sebastiani G, Alberti A. Non invasive fibrosis biomarkers reduce but not substitute the need for liver biopsy. World I Gastroenterol 2006:12:3682-3694.

8. Ophir J, Cespedes I, Ponnekanti H, Yazdi Y, Li X. Elastography: a quantitative method for imaging the elasticity of biological tissues. Ultrason Imaging 1991;13:111-134.

9. Mariappan YK, Glaser KJ, Ehman RL. Magnetic resonance elastography: a review. Clin Anat 2010;23:497-511.

10. Nightingale K. Acoustic radiation force impulse (ARFI) imaging: a review. Curr Med Imaging Rev 2011;7:328-339.

11. Bercoff J, Tanter M, Fink M. Supersonic shear imaging: a new technique for soft tissue elasticity mapping. IEEE Trans Ultrason Ferroelectr Freq Control 2004;51:396-409.

12. Koizumi Y, Hirooka M, Kisaka Y, Konishi I, Abe M, Murakami H, et al. Liver fibrosis in patients with chronic hepatitis C: noninvasive diagnosis by means of real-time tissue elastography--establishment of the method for measurement. Radiology 2011;258:610-617.

13. Friedrich-Rust M, Ong MF, Herrmann E, Dries V, Samaras $P$, Zeuzem $S$, et al. Real-time elastography for noninvasive assessment of liver fibrosis in chronic viral hepatitis. AJR Am J Roentgenol 2007; 188:758-764.

14. Sandrin L, Fourquet B, Hasquenoph JM, Yon S, Fournier C, Mal $F$, et al. Transient elastography: a new noninvasive method for assessment of hepatic fibrosis. Ultrasound Med Biol 2003;29:17051713. 
15. Krouskop TA, Dougherty DR, Vinson FS. A pulsed Doppler ultrasonic system for making noninvasive measurements of the mechanical properties of soft tissue. J Rehabil Res Dev 1987;24:1-8.

16. Barannik EA, Girnyk A, Tovstiak V, Marusenko Al, Emelianov SY, Sarvazyan AP. Doppler ultrasound detection of shear waves remotely induced in tissue phantoms and tissue in vitro. Ultrasonics 2002;40:849-852.

17. Kim SU, Seo YS, Cheong JY, Kim MY, Kim JK, Um SH, et al. Factors that affect the diagnostic accuracy of liver fibrosis measurement by Fibroscan in patients with chronic hepatitis B. Aliment Pharmacol Ther 2010;32:498-505.

18. Foucher J, Castera L, Bernard PH, Adhoute X, Laharie D, Bertet J, et al. Prevalence and factors associated with failure of liver stiffness measurement using FibroScan in a prospective study of 2114 examinations. Eur I Gastroenterol Hepatol 2006;18:411-412.

19. Friedrich-Rust M, Wunder K, Kriener S, Sotoudeh F, Richter S, Bojunga J, et al. Liver fibrosis in viral hepatitis: noninvasive assessment with acoustic radiation force impulse imaging versus transient elastography. Radiology 2009;252:595-604.

20. Bavu E, Gennisson JL, Couade M, Bercoff J, Mallet V, Fink M, et al. Noninvasive in vivo liver fibrosis evaluation using supersonic shear imaging: a clinical study on 113 hepatitis C virus patients. Ultrasound Med Biol 2011;37:1361-1373.

21. Cha SW, Jeong WK, Kim Y, Kim MY, Kim J, Kim SY, et al. Nondiseased liver stiffness measured by shear wave elastography: a pilot study. J Ultrasound Med 2014;33:53-60.

22. Poynard T, Munteanu M, Luckina E, Perazzo H, Ngo Y, Royer L, et al. Liver fibrosis evaluation using real-time shear wave elastography: applicability and diagnostic performance using methods without a gold standard. J Hepatol 2013;58:928-935.

23. Stebbing J, Farouk L, Panos G, Anderson M, Jiao LR, Mandalia S, et al. A meta-analysis of transient elastography for the detection of hepatic fibrosis. J Clin Gastroenterol 2010;44:214-219.

24. Tsochatzis EA, Gurusamy KS, Ntaoula S, Cholongitas E, Davidson $B R$, Burroughs AK. Elastography for the diagnosis of severity of fibrosis in chronic liver disease: a meta-analysis of diagnostic accuracy. J Hepatol 2011;54:650-659.

25. Boursier J, Isselin G, Fouchard-Hubert I, Oberti F, Dib N, Lebigot $J$, et al. Acoustic radiation force impulse: a new ultrasonographic technology for the widespread noninvasive diagnosis of liver fibrosis. Eur I Gastroenterol Hepatol 2010;22:1074-1084.

26. Friedrich-Rust M, Nierhoff J, Lupsor M, Sporea I, FierbinteanuBraticevici C, Strobel D, et al. Performance of Acoustic Radiation Force Impulse imaging for the staging of liver fibrosis: a pooled meta-analysis. J Viral Hepat 2012;19:e212-e219.

27. Marcellin P, Ziol M, Bedossa P, Douvin C, Poupon R, de Ledinghen $V$, et al. Non-invasive assessment of liver fibrosis by stiffness measurement in patients with chronic hepatitis B. Liver Int 2009;29:242-247.
28. Leroy V, Kim SU. Can transient elastography be used for the management of chronic hepatitis B patients? Liver Int 2012;32:528-530.

29. Chon YE, Choi EH, Song KJ, Park JY, Kim do Y, Han KH, et al. Performance of transient elastography for the staging of liver fibrosis in patients with chronic hepatitis B: a meta-analysis. PLoS One 2012; 7:e44930.

30. Oliveri F, Coco B, Ciccorossi P, Colombatto P, Romagnoli V, Cherubini $B$, et al. Liver stiffness in the hepatitis $B$ virus carrier: a non-invasive marker of liver disease influenced by the pattern of transaminases. World J Gastroenterol 2008;14:6154-6162.

31. Matteoni CA, Younossi ZM, Gramlich T, Boparai N, Liu YC, McCullough AJ. Nonalcoholic fatty liver disease: a spectrum of clinical and pathological severity. Gastroenterology 1999; 116:1413-1419.

32. Sasso M, Beaugrand $M$, de Ledinghen $V$, Douvin $C$, Marcellin $P$, Poupon $\mathrm{R}$, et al. Controlled attenuation parameter (CAP): a novel VCTETM guided ultrasonic attenuation measurement for the evaluation of hepatic steatosis: preliminary study and validation in a cohort of patients with chronic liver disease from various causes. Ultrasound Med Biol 2010;36:1825-1835.

33. Cassinotto C, Lapuyade B, Ait-Ali A, Vergniol J, Gaye D, Foucher J, et al. Liver fibrosis: noninvasive assessment with acoustic radiation force impulse elastography--comparison with FibroScan $\mathrm{M}$ and $\mathrm{XL}$ probes and FibroTest in patients with chronic liver disease. Radiology 2013;269:283-292.

34. Festi D, Schiumerini R, Marzi L, Di Biase AR, Mandolesi D, Montrone $L$, et al. Review article: the diagnosis of non-alcoholic fatty liver disease: availability and accuracy of non-invasive methods. Aliment Pharmacol Ther 2013;37:392-400.

35. Wong VW, Vergniol J, Wong GL, Foucher J, Chan HL, Le Bail B, et al. Diagnosis of fibrosis and cirrhosis using liver stiffness measurement in nonalcoholic fatty liver disease. Hepatology 2010;51:454-462.

36. Adebajo CO, Talwalkar JA, Poterucha JJ, Kim WR, Charlton MR. Ultrasound-based transient elastography for the detection of hepatic fibrosis in patients with recurrent hepatitis $C$ virus after liver transplantation: a systematic review and meta-analysis. Liver Transpl 2012;18:323-331.

37. Yoon JH, Lee JY, Woo HS, Yu MH, Lee ES, Joo I, et al. Shear wave elastography in the evaluation of rejection or recurrent hepatitis after liver transplantation. Eur Radiol 2013;23:1729-1737.

38. Robic MA, Procopet B, Metivier S, Peron JM, Selves J, Vinel JP, et al. Liver stiffness accurately predicts portal hypertension related complications in patients with chronic liver disease: a prospective study. J Hepatol 2011;55:1017-1024.

39. Vizzutti F, Arena U, Romanelli RG, Rega L, Foschi M, Colagrande $S$, et al. Liver stiffness measurement predicts severe portal hypertension in patients with HCV-related cirrhosis. Hepatology 2007;45:1290-1297. 
40. Kim BK, Fung J, Yuen MF, Kim SU. Clinical application of liver stiffness measurement using transient elastography in chronic liver disease from longitudinal perspectives. World I Gastroenterol 2013;19:1890-1900.

41. Fung J, Lai CL, Wong DK, Seto WK, Hung I, Yuen MF. Significant changes in liver stiffness measurements in patients with chronic hepatitis B: 3-year follow-up study. J Viral Hepat 2011;18: e200-e205.

42. Vergniol J, Foucher J, Castera L, Bernard PH, Tournan R, Terrebonne $E$, et al. Changes of non-invasive markers and FibroScan values during HCV treatment. J Viral Hepat 2009;16:132-140.

43. Kim BK, Han KH, Park JY, Ahn SH, Kim JK, Paik YH, et al. A liver stiffness measurement-based, noninvasive prediction model for high-risk esophageal varices in B-viral liver cirrhosis. Am J Gastroenterol 2010;105:1382-1390.

44. Kim BK, Kim do Y, Han KH, Park JY, Kim JK, Paik YH, et al. Risk assessment of esophageal variceal bleeding in B-viral liver cirrhosis by a liver stiffness measurement-based model. Am J Gastroenterol 2011;106:1654-1662.

45. Fraquelli M, Rigamonti C, Casazza G, Conte D, Donato MF, Ronchi $\mathrm{G}$, et al. Reproducibility of transient elastography in the evaluation of liver fibrosis in patients with chronic liver disease. Gut 2007;56:968-973.

46. Boursier J, Konate A, Guilluy M, Gorea G, Sawadogo A, Quemener $E$, et al. Learning curve and interobserver reproducibility evaluation of liver stiffness measurement by transient elastography. Eur J Gastroenterol Hepatol 2008;20:693-701.

47. Arena U, Vizzutti F, Corti G, Ambu S, Stasi C, Bresci S, et al. Acute viral hepatitis increases liver stiffness values measured by transient elastography. Hepatology 2008;47:380-384.
48. Millonig $G$, Reimann FM, Friedrich $S$, Fonouni $H$, Mehrabi A, Buchler MW, et al. Extrahepatic cholestasis increases liver stiffness (FibroScan) irrespective of fibrosis. Hepatology 2008;48:17181723.

49. Millonig $G$, Friedrich $S$, Adolf $S$, Fonouni $H$, Golriz M, Mehrabi $A$, et al. Liver stiffness is directly influenced by central venous pressure. J Hepatol 2010;52:206-210.

50. Mederacke I, Wursthorn K, Kirschner J, Rifai K, Manns MP, Wedemeyer $\mathrm{H}$, et al. Food intake increases liver stiffness in patients with chronic or resolved hepatitis $C$ virus infection. Liver Int 2009;29:1500-1506.

51. Bota S, Sporea I, Sirli R, Popescu A, Danila M, Costachescu D. Intraand interoperator reproducibility of acoustic radiation force impulse (ARFI) elastography: preliminary results. Ultrasound Med Biol 2012;38:1103-1108.

52. Ferraioli G, Tinelli C, Zicchetti M, Above E, Poma G, Di Gregorio M, et al. Reproducibility of real-time shear wave elastography in the evaluation of liver elasticity. Eur J Radiol 2012;81:3102-3106.

53. Yoon JH, Lee JM, Woo HS, Yu MH, Joo I, Lee ES, et al. Staging of hepatic fibrosis: comparison of magnetic resonance elastography and shear wave elastography in the same individuals. Korean $\mathrm{J}$ Radiol 2013;14:202-212.

54. Bamber J, Cosgrove D, Dietrich CF, Fromageau J, Bojunga J, Calliada $F$, et al. EFSUMB guidelines and recommendations on the clinical use of ultrasound elastography. Part 1: Basic principles and technology. Ultraschall Med 2013;34:169-184.

55. Cosgrove D, Piscaglia F, Bamber J, Bojunga J, Correas JM, Gilja $\mathrm{OH}$, et al. EFSUMB guidelines and recommendations on the clinical use of ultrasound elastography. Part 2: Clinical applications. Ultraschall Med 2013;34:238-253. 